\title{
OPEN Differential relationships of PTSD symptom clusters with cortical thickness and grey matter volumes among women with PTSD
}

\author{
Kevin M. Crombie ${ }^{\bowtie}$, Marisa C. Ross, Allison M. Letkiewicz, Anneliis Sartin-Tarm \& \\ Josh M. Cisler
}

Structural neuroimaging studies of posttraumatic stress disorder (PTSD) have typically reported reduced cortical thickness (CT) and gray matter volume (GMV) in subcortical structures and networks involved in memory retrieval, emotional processing and regulation, and fear acquisition and extinction. Although PTSD is more common in women, and interpersonal violence (IPV) exposure is a more potent risk factor for developing PTSD relative to other forms of trauma, most of the existing literature examined combat-exposed men with PTSD. Vertex-wise CT and subcortical GMV analyses were conducted to examine potential differences in a large, well-characterized sample of women with PTSD stemming from IPV-exposure $(n=99)$ compared to healthy trauma-free women without a diagnosis of PTSD $(n=22)$. Subgroup analyses were also conducted to determine whether symptom severity within specific PTSD symptom clusters (e.g., re-experiencing, active avoidance, hyperarousal) predict CT and GMV after controlling for comorbid depression and anxiety. Results indicated that a diagnosis of PTSD in women with IPV-exposure did not significantly predict differences in CT across the cortex or GMV in the amygdala or hippocampus compared to healthy controls. However, within the PTSD group, greater re-experiencing symptom severity was associated with decreased CT in the left inferior and middle temporal gyrus, and decreased CT in the right parahippocampal and medial temporal gyrus. In contrast, greater active avoidance symptom severity was associated with greater CT in the left lateral fissure, postcentral gyrus, and middle/lateral occipital cortex, and greater CT in the right paracentral, posterior cingulate, and superior occipital gyrus. In terms of GMV, greater hyperarousal symptom severity was associated with reduced left amygdala GMV, while greater active avoidance symptom severity was associated with greater right amygdala GMV. These findings suggest that structural brain alterations among women with IPV-related PTSD may be driven by symptom severity within specific symptom clusters and that PTSD symptom clusters may have a differential (increased or decreased) association with brain structures.

Posttraumatic stress disorder (PTSD) is a highly debilitating mental health disorder characterized by intrusive recollection of traumatic memories, avoidance of people and places that trigger recall of traumatic memories, and prolonged physiological hyperarousal and vigilance towards danger ${ }^{1}$. Although individuals can develop PTSD following exposure to a broad range of traumatic events (e.g., combat, motor vehicle accidents), epidemiological evidence suggests that interpersonal violence (IPV) exposure (i.e., physical or sexual assault) is a more potent risk factor than other forms of trauma ${ }^{2-4}$. PTSD, which is more common in women than $m^{5-7}$, is associated with markedly elevated morbidity, distress, and decreased quality of life relative to other anxiety-related disorders ${ }^{8}$. Psychotherapeutic approaches (e.g., exposure-based therapy) are among the best supported interventions for PTSD, yet remission rates for these front-line treatments are typically only $50-60 \%{ }^{9,10}$. Thus, a better mechanistic understanding of PTSD across various levels (e.g., neural, molecular, cellular) and modalities (e.g., neuroimaging) may contribute to the development or enhancement of novel or existing therapies, or potentially elucidate why certain therapies have yielded sub-optimal efficacy rates.

Advances in functional and structural neuroimaging techniques and computational analytic approaches has led to an improved understanding of neural correlates of PTSD. Much of our understanding of the structural 
correlates of PTSD stems from studies examining subcortical gray matter volume (GMV) and structural alterations in cortical thickness (CT) among combat-exposed men with PTSD. For instance, Bremner et al. provided the first evidence of a significant reduction in hippocampal GMV of patients with combat related PTSD compared to age and sex-matched healthy controls ${ }^{11}$, a finding that has since been replicated in this population ${ }^{12-15}$. Since this seminal study, numerous independent investigations and meta-analytic evidence largely supports the notion that a diagnosis of PTSD stemming from several sources of trauma (e.g., combat-related trauma, motor vehicle accidents, natural disaster survivors) is associated with reduced GMV in subcortical structures and reduced $\mathrm{CT}$ in several regions, including but not limited to the amygdala, prefrontal cortex (medial, ventral, dorsolateral), inferior, superior, postcentral, and middle temporal gyri, and anterior and posterior cingulate cortex ${ }^{16-27}$. Accordingly, deficits in CT and subcortical volumes within this population are present in regions and networks involved in memory retrieval, emotional processing and regulation, decision-making, and fear acquisition and extinction, all processes known to be altered in PTSD $^{28-30}$.

In contrast, there is a scarcity of investigations examining potential differences in GMV and CT in adult women with PTSD specifically resulting from IPV-exposure, although there is a recent increase in research examining women with PTSD in general ${ }^{31,32}$. The lack of research within this population is unfortunate as: (1) women are more than twice as likely to develop a diagnosis of PTSD following trauma exposure ${ }^{5-7}$, and (2) the probability of developing PTSD is greatest following IPV exposure, which is more common in women ${ }^{4,7,33-35}$. Given known sex differences in PTSD in prevalence, exposure to physical and sexual assault, and comorbid depression and anxiety (all with greater rates in women), it is important to probe structural correlates among women with PTSD specifically ${ }^{4-7,33-36}$. One of the few investigations conducted to date examined a small sample of women experiencing chronic PTSD $(n=17)$ following sexual abuse and found no difference in global and regional brain volumes and CT compared to age and education-matched controls ${ }^{37}$. Relatedly, an investigation of women $(n=11)$ with interpersonal partner violence histories ${ }^{38}$ found no evidence of gray matter deficits within subcortical structures (e.g., hippocampus), which has been commonly reported in male samples with combat related PTSD ${ }^{11,13-15}$. In contrast, a small sample $(n=15)$ consisting primarily of women with PTSD stemming from non-combat related traumatic experiences (66\% of sample consisted of women, $87 \%$ of sample experienced IPV) reported a positive relationship between avoidance symptoms and right amygdala and hippocampal volume ${ }^{39}$. Additionally, literature examining childhood trauma (which incorporates interpersonal trauma) and $\mathrm{CT}$ has yielded equivocal findings as a recent report ${ }^{40}$ indicated that adolescents with childhood sexual abuse (85\% of sample consisted of females) preserved CT (in all a priori regions of interest), while other investigations (consisting of both adolescents and adult males and females) have reported altered CT throughout the brain ${ }^{41-43}$. Overall, our understanding of potential differences in GMV and CT in adult women with PTSD resulting from IPV-exposure could benefit from investigations with larger sample sizes.

Another important consideration that has not been thoroughly examined within this population is differentiating structural correlates of PTSD from depression and general anxiety. Meta-analytic evidence indicates that depressed individuals often exhibit structural differences (e.g., decreased GMV within subcortical limbic brain regions) similar to those commonly reported among individuals with PTSD $^{44-46}$. Given that depression and anxiety are highly comorbid in women with IPV-related PTSD ${ }^{7,47-49}$, it is important for investigations to also include and control for depression and anxiety in structural analyses, so that we are better able to identify the unique contribution of PTSD. Relatedly, previous investigations have primarily focused on overall PTSD symptom severity, while neglecting to examine whether symptom severity within specific PTSD symptom clusters predicts different structural correlates. Examining such relationships could prove important as differences in symptom severity within clusters has previously been found to differentially influence interpersonal functioning, physical health, and comorbid mental health diagnoses, including depression ${ }^{50,51}$.

Given the differential structural findings (i.e., a potential lack of structural differences in women with IPVexposure) compared to primarily male combat veteran populations, the limited existing data highlight the importance of further examining the neural mechanisms of physical and sexual assault-related PTSD in larger samples of women, for which the traumatic experience is vastly different. Furthermore, the association between CT, GMV, and PTSD symptom severity (for each symptom cluster) within a sample of women with IPV-related PTSD, to our knowledge, has not yet been investigated. As a result, the current study sought to conduct vertexwise CT and subcortical GMV analyses to examine potential differences in a large, sample of adult women with PTSD stemming from IPV-exposure $(\mathrm{n}=99)$ compared to non-trauma exposed adult women without a diagnosis of PTSD $(n=22)$. Given the substantial symptom heterogeneity in PTSD, this study also sought to move beyond a simple dichotomized comparison (PTSD vs. no PTSD) by conducting subgroup analyses to determine whether symptom severity within specific PTSD symptom clusters (e.g., re-experiencing, avoidance, hyperarousal) predicts CT and GMV in adult women with PTSD from IPV-exposure. Based on prior investigations (primarily examining men) and meta-analytic evidence ${ }^{11-27,39}$, it was hypothesized that (1) women with PTSD stemming from IPV-exposure would exhibit reduced amygdala and hippocampal GMV, and reduced CT in several regions, including: prefrontal cortex (medial, ventral, dorsolateral), inferior, superior, postcentral, and middle temporal gyri, and anterior and posterior cingulate cortex compared to non-trauma exposed women without a diagnosis of PTSD; and (2) among women with PTSD, greater avoidance symptom severity scores would predict greater right amygdala and hippocampal volume.

\section{Materials and methods}

The work described in this manuscript has been carried out in accordance with The Code of Ethics of the World Medical Association (Declaration of Helsinki) for experiments involving humans, and all subjects completed informed consent. 
Participant recruitment. Adult females aged 21-50 were recruited through three different studies conducted at two sites: the University of Arkansas for Medical Sciences (UAMS) and the University of WisconsinMadison (UW). All study procedures were approved by the Institutional Review Board at UAMS and the UW Health Sciences Institutional Review Board, and all methods were carried out in accordance with relevant guidelines and regulations. The analyses included in the current manuscript are independent of previously reported findings $s^{52-55}$. These studies recruited women with a primary diagnosis of PTSD and a primary index trauma of IPV-exposure, along with healthy women, free of trauma exposure or mental health concerns. Inclusion criteria for the PTSD group in all studies was a primary diagnosis of PTSD, IPV-exposure, and female sex. Inclusion criteria for the healthy control groups included female sex, absence of trauma exposure, absence of current psychiatric disorders, and absence of psychiatric medication use. Exclusion criteria for all participants included internal metal or other MRI contraindications, major medical disorders, and endorsement of psychotic symptoms.

Assessments. Participants completed a baseline demographic questionnaire assessing age, race, and education. Trauma histories were assessed with the National Women's Survey (NSA) ${ }^{56}$ trauma assessment section, which is a structured interview that assesses previous history of physical abuse by a caregiver, physical assault, sexual assault, witnessed domestic violence, witnessed community violence, and a range of other stressful life events. The presence of mental health and psychiatric disorders (e.g., major depressive disorder, anxiety disorders) was assessed using the Structured Clinical Interview for DSM-IV ${ }^{57}$. PTSD diagnosis and symptom severity was assessed using the Clinician Administered PTSD Scale for DSM-5 (CAPS-5) ${ }^{58}$ and the PTSD Checklist for DSM-5 (PCL-5) $)^{59}$ for two studies $(\mathrm{n}=110)$ and the Structured Clinical Interview for DSM-IV Disorders (SCIDIV) and PTSD Checklist-Civilian Version $(\text { PCL-C })^{60}$ for one study $(n=30)$. Of the 121 participants included in the analyses, 11 participants (50\%) from the control group and 15 participants (15\%) from the PTSD group completed DSM-IV measures, while 11 participants (50\%) from the control group and 84 participants (85\%) from the PTSD group completed DSM-V measures. PCL-5 scores were used as the measure of PTSD symptom severity for each cluster and for overall symptom severity in all analyses including PTSD symptoms. As a result, PCL-C scores were converted to PCL-5 scores using an established and validated crosswalk procedure using equipercentile equating ${ }^{61}$. All interview-based assessments were conducted by trained clinical interviewers. See supplementary material for correlation table of assessment factors.

Data acquisition. At the UAMS site $(\mathrm{n}=72)$, T1-weighted anatomic images were acquired with an MPRAGE sequence (matrix $=192 \times 192,160$ sagittal slices, TR/TE/FA $=7.5 / 3.7 / 9^{\circ}$, FOV $=256,256,160$, final resolution $=1 \times 1 \times 1 \mathrm{~mm}$ ) on a Philips Achieva $3 \mathrm{~T}$ X-Series scanner with a 32 -channel headcoil. At the UW site $(n=68)$, T1-weighted anatomic images were acquired with a similar MP-RAGE sequence (matrix $=256 \times 256$, 156 axial slices, $\mathrm{TR} / \mathrm{TE} / \mathrm{FA}=8.2 \mathrm{~ms} / 3.2 \mathrm{~ms} / 12^{\circ}, \mathrm{FOV}=25.6 \mathrm{~cm}$, final resolution $\left.=1 \times 1 \times 1 \mathrm{~mm}\right)$ on a GE MR750 $3 \mathrm{~T}$ scanner with an 8-channel headcoil. See supplementary material for additional information regarding group summaries for each scanning site.

Quality control and attrition. All T1-weighted images were visually inspected to identify and exclude poor quality images. Following inspection, 17 images were excluded due to poor quality $\mathrm{T} 1 \mathrm{~s}$, due to participant head movement $(\mathrm{n}=16)$, and a neuroanatomical anomaly $(\mathrm{n}=1)$. Additionally, two individuals had high quality T1 images, but were missing PCL-5 scores. The remaining 121 raw T1-images were retained and consisted of the final sample for data analyses.

Image preprocessing. Preprocessing of the raw T1-weighted images was completed in FreeSurfer Version 6.0.0 (http://surfer.nmr.mgh.harvard.edu) ${ }^{62}$ on a Linux platform. FreeSurfer's reconall command was used to preprocess all images through standard steps, including motion correction and averaging ${ }^{63}$, removal of nonbrain tissue and skull-stripping ${ }^{64}$, automated Talariach transformation, segmentation of subcortical white and gray matter ${ }^{62,63}$, transformation to MNI305 atlas space, intensity normalization ${ }^{65}$, and volumetric registration. Technical details for FreeSurfer are described in detail elsewhere ${ }^{62-64,66-72}$.

Image analysis. For all images, reconall continued without intervention through the processing stream following initial preprocessing steps. This included steps for another intensity normalization, white matter segmentation and editing, tessellation of white and gray matter boundaries and automated topology correction ${ }^{73,68}$, smoothing and surface inflation, spherical mapping and registration for each hemisphere ${ }^{67,68,70}$, and parcellation and labelling of cortical and subcortical structures. Cortical structures were labelled according to the DesikanKilliany-Tourville parcellation (DKT $)^{74}$ and mapped to the spherical surface, while subcortical and white matter structures are labelled according to FreeSurfer's automated segmentation tool (aseg) $)^{58}$ and mapped to the MNI305 common space. Final statistics for GMV, CT, and surface area estimates of each region by hemisphere in both the DKT and subcortical aseg parcellations were generated.

Vertex-wise cortical thickness analysis. Two separate vertex-wise CT analyses were conducted to: (1) examine group differences between control and PTSD groups; and (2) examine whether PTSD symptom severity scores for each PTSD symptom cluster are associated with CT in the PTSD group only. To conduct these vertex-wise analyses of potential differences in CT across the brain, we first generated cortical surface files for each subject. Subject surface files were registered to the average template (fsaverage), which is constructed from scans of 40 healthy adult subjects ${ }^{75}$ and smoothed with a $15 \mathrm{~mm}$ full width half-maximum kernel in order to compare differences in CT across subjects. Next, vertex-wise linear model tests across the cortical surface were 


\begin{tabular}{|c|c|c|}
\hline & Control $(n=22)$ & PTSD $(\mathbf{n}=99)$ \\
\hline Age (years) & $31.68 \pm 7.92$ & $33.48 \pm 8.48$ \\
\hline Education (years) & $16.73 \pm 2.47^{\star}$ & $15.00 \pm 2.51$ \\
\hline \multicolumn{3}{|l|}{ Race } \\
\hline$\#(\%)$, Caucasian & $17(77.27)$ & $72(72.72)$ \\
\hline \#(\%), Black/African-American & $3(13.63)$ & $18(18.18)$ \\
\hline \#(\%), Hispanic/Latino & $2(9.09)$ & $2(2.02)$ \\
\hline$\#(\%)$, Asian & $0(0)$ & $1(1.01)$ \\
\hline \#(\%), Native American & $0(0)$ & $1(1.01)$ \\
\hline$\#(\%)$, other & $0(0)$ & $5(5.05)$ \\
\hline $\operatorname{TIV}\left(\mathrm{mm}^{3} \times 10^{6}\right)$ & $1.44 \pm 0.11$ & $1.40 \pm 0.12$ \\
\hline \multicolumn{3}{|l|}{ PTSD symptom severity } \\
\hline Re-experiencing & - & $10.80 \pm 4.75$ \\
\hline Avoidance & - & $5.38 \pm 2.03$ \\
\hline Negative alterations in cognition $/$ mood & - & $16.12 \pm 6.10$ \\
\hline Hyperarousal/reactivity & - & $12.15 \pm 4.90$ \\
\hline Overall & - & $44.57 \pm 14.95$ \\
\hline Trauma exposure (\# of direct assaults experienced) & - & $5.75 \pm 2.93$ \\
\hline Current major depressive disorder, \# (\%) & - & $30(30.30)$ \\
\hline Current anxiety disorder, \# (\%) & - & $67(67.67)$ \\
\hline
\end{tabular}

Table 1. Participant characteristics. Values listed as $M \pm S D$ unless otherwise noted. ${ }^{*}$ indicates significant $(p<.05)$ group difference. TIV = estimated total intracranial volume.

conducted using either group (control vs PTSD) or PTSD symptom severity scores for PTSD symptom clusters (i.e., re-experiencing, active avoidance, negative alterations in cognition and mood, and hyperarousal) as the main predictor variables for each separate set of analyses (i.e., group [PTSD vs control group] analysis, and PTSD symptom cluster analysis). Additionally, given that the control group consisted of non-trauma exposed women, exploratory analyses (see supplementary material) were conducted examining trauma exposure (i.e., number of IPV-related direct assaults experienced) as a main predictor of interest (as opposed to group: control vs PTSD). All comparisons were conducted using AFNI's 3dttest ++ function $^{76}$, controlling for participant age, image acquisition site (UW or UAMS), and current diagnoses of major depression or anxiety disorders. To control for multiple comparisons, we used the AFNI slow_surf_clustsim.py function to run clustsim on the grouplevel fsaverage surface. We specified the uncorrected $p$ threshold at 0.001 and ran 10,000 Monte Carlo simulations to generate the minimum surface cluster area unlikely to be detected due to chance $(\mathrm{p}>0.05)$. Finally, cortical regions with thickness that significantly related to either group or PTSD symptom severity for each PTSD symptom cluster above the area threshold were extracted using AFNI SurfClust.

Gray matter region of interest selection. Gray matter regions of interest (ROIs) included the hippocampus and amygdala of both the left and right hemispheres. ROIs were selected a priori based on previous research suggesting reduced volume in PTSD as a result of various trauma exposures ${ }^{22-24}$.

Gray matter data analysis. Following processing in Freesurfer, gray matter ROIs from the DKT parcellation were entered into multiple linear regression models. Two sets of models were tested for each hemisphere for both ROIs (amygdala and hippocampus). Each model started off including covariates of non-interest (age, site, estimated total intercranial volume [eTIV]), before adding the main predictors of interest (i.e., group, PTSD cluster symptom severity scores, respectively for each set of models), followed by the inclusion of additional covariates (i.e., current diagnosis of anxiety disorder and major depressive disorder, coded as separate variables) that are prevalent within PTSD, and therefore may explain unique variance. The model examining group differences in GMV used dummy-coded contrasts with the control group as the reference group. Models were estimated using the fitlm function in Matlab. In order to avoid an inflated Type 1 error rate, we corrected for multiple comparisons by dividing the $p$-value of 0.05 by $4(p=0.0125)$. Lastly, given that the control group consisted of non-trauma exposed women, exploratory analyses (see supplementary material) were conducted examining trauma exposure (i.e., number of IPV-related direct assaults experienced) as a main predictor of interest (as opposed to group: control vs PTSD).

\section{Results}

Participant characteristics. See Table 1 for a complete description of participant characteristics. Independent samples t-tests indicated that groups did not significantly differ in age $(t(119)=0.91, p=0.364)$ or eTIV $(t(119)=-1.44, p=0.151)$. The control group had significantly greater number of years of education compared to the PTSD group $(t(119)=-2.92, p=0.004)$. 
A
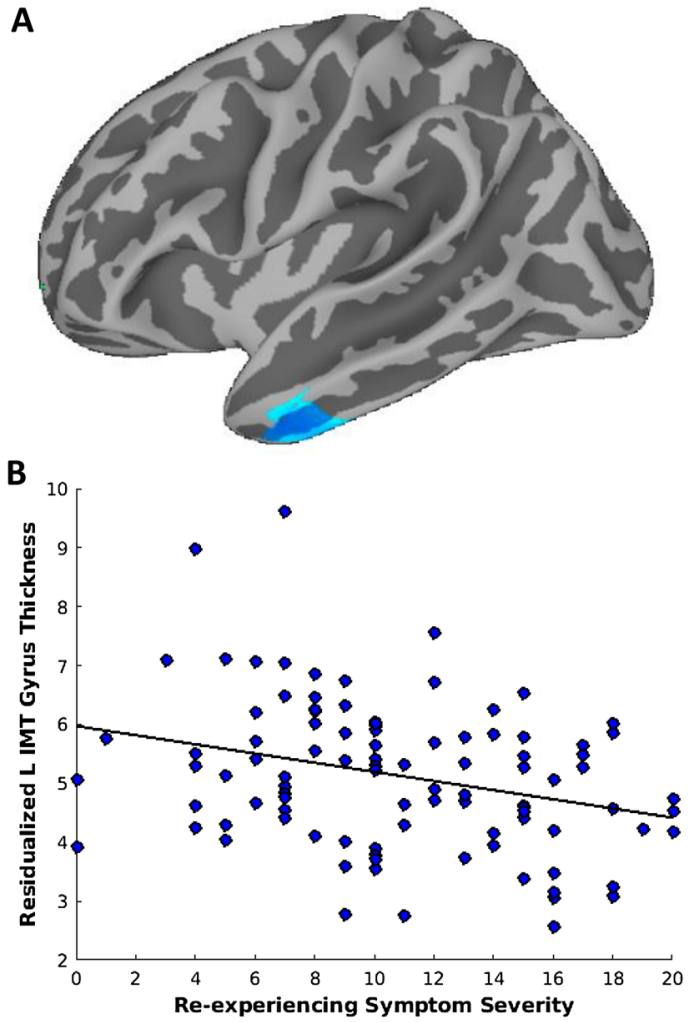

C
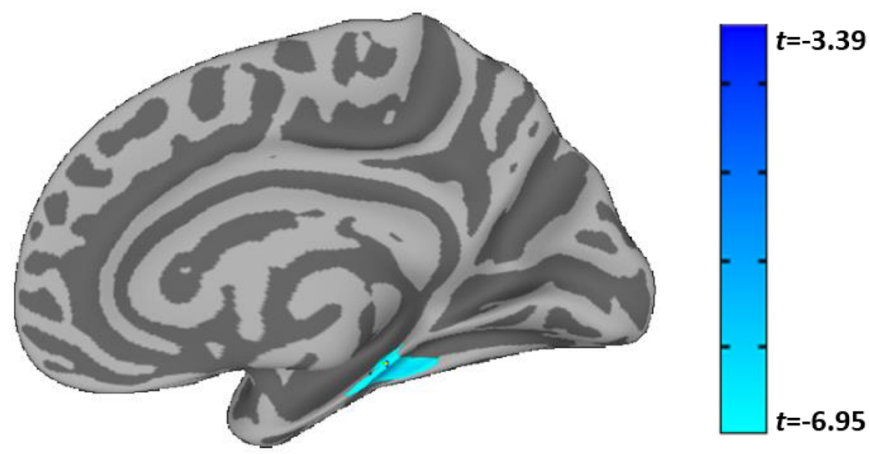

D

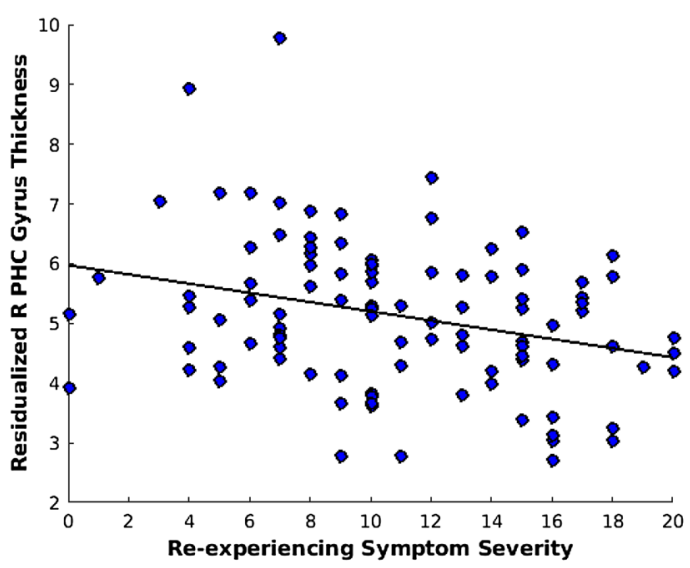

Figure 1. Greater Re-experiencing PTSD symptom severity is associated with decreased thickness of the left inferior and middle temporal gyrus and right parahippocampal gyrus. Vertex-wise linear model tests across the cortical surface revealed two clusters that survived Monte-Carlo correction for multiple comparisons and were associated with greater PTSD re-experiencing symptom severity: the left inferior and middle temporal gyrus $(\mathbf{A}, \mathbf{B})$ and the right parahippocampal gyrus $(\mathbf{C}, \mathbf{D})$. Re-experiencing symptom severity scores (cluster B; score range of 0-20) were obtained from the PTSD Checklist for DSM-V (PCL-5). PTSD = posttraumatic stress disorder; $\mathrm{L}=$ left; $\mathrm{R}=$ right; $\mathrm{IMT}=$ inferior/middle temporal; $\mathrm{PHC}=$ parahippocampal.

Cortical thickness. Control versus PTSD. A diagnosis of PTSD did not significantly ( $p>0.05$ corrected for whole brain comparison) predict differences in CT across the entire cortical surface after controlling for age, scanner site, anxiety, and depression.

PTSD symptom clusters. Within the PTSD group, greater re-experiencing symptoms were significantly associated with decreased $\mathrm{CT}$ in the left inferior and middle temporal gyrus (Fig. 1A,B) and decreased CT in the right parahippocampal and medial temporal gyrus (Fig. 1C,D). Greater active avoidance symptoms were significantly associated with greater CT in the left lateral fissure (Fig. 2A,B), postcentral gyrus (Fig. 2C,D), and middle/lateral occipital gyrus (Fig. 2E,F) and greater CT in the right paracentral (Fig. 3A,B), posterior cingulate, and superior occipital gyrus (Fig. 3C,D). Symptoms of hyperarousal and negative alterations in cognition and mood did not significantly ( $p>0.05$ corrected for whole brain comparison) predict differences in CT across the entire cortical surface after controlling for age, scanner site, anxiety, and depression.

Volumetric analyses. Control versus PTSD. A diagnosis of PTSD did not significantly predict differences in amygdala (left and right, $p s=0.739$ and 0.636 , respectively) or hippocampal (left and right, $p s=0.696$ and 0.337, respectively) volume (see Table 2; Fig. 4A,B, respectively). There was a trend toward a current diagnosis of major depressive disorder predicting less right amygdala volume $(p=0.020$; see Table 2$)$.

PTSD symptom clusters. Within the PTSD group, greater hyperarousal symptoms were significantly $(p=0.007)$ associated with less left amygdala volume, even after controlling for anxiety and depression (see Table 3; Fig. 4C). Greater active avoidance symptoms were significantly $(p=0.012)$ associated with greater right amygdala volume, even after controlling for depression and anxiety (see Table 3; Fig. 4D). Re-experiencing and negative alterations in cognition and mood symptoms did not significantly predict left ( $p s=0.923$ and 0.757 , respectively) or right ( $p s=0.424$ and 0.803 , respectively) amygdala volume. PTSD symptom cluster scores did not significantly predict left ( $p s=0.134$ to 0.647 ) or right $(p s=0.338$ to 0.996$)$ hippocampal volume (see Table 3 ). 

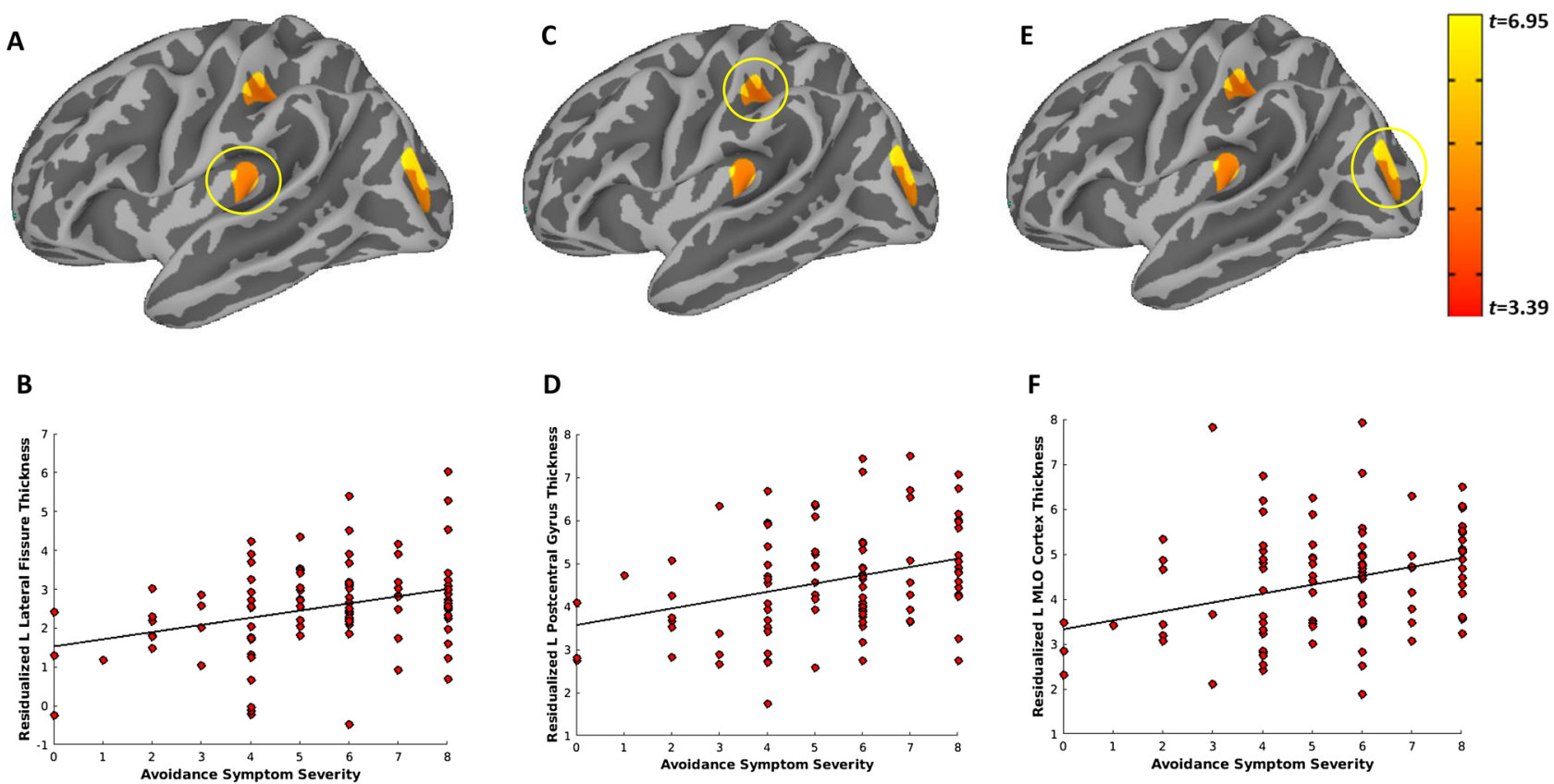

Figure 2. Greater Active Avoidance PTSD symptom severity is associated with increased thickness of the left lateral fissure, postcentral gyrus, and middle/lateral occipital cortex. Vertex-wise linear model tests across the cortical surface revealed three clusters that survived Monte-Carlo correction for multiple comparisons and were associated with greater PTSD re-avoidance symptom severity: the left lateral fissure (A,B), postcentral gyrus $(\mathbf{C}, \mathbf{D})$, middle/lateral occipital cortex (E,F). Active Avoidance symptom severity scores (cluster C; score range of 0-8) were obtained from the PTSD Checklist for DSM-V (PCL-5). PTSD = posttraumatic stress disorder. L= left; $\mathrm{MLO}=$ middle/lateral occipital.

\section{Discussion}

This study investigated CT and subcortical GMV among adult women with PTSD resulting from IPV-exposure and a group of healthy, age-matched women free of trauma exposure or mental health concerns. Results from the current study revealed that a diagnosis of PTSD among IPV-exposed women did not significantly predict differences in CT across the cortex or GMV in the amygdala or hippocampus compared to trauma-free women without PTSD. However, re-experiencing, active avoidance, and hyperarousal symptom severity within the PTSD group were significantly associated with differential (i.e., increased and decreased) CT in several regions and GMV in the amygdala.

Previous research among adults with PTSD, mostly male veterans, has consistently demonstrated reduced CT in several regions including the anterior cingulate cortex, prefrontal cortex (medial, ventral, dorsolateral), and inferior and superior temporal gyri ${ }^{17,19,20,23,77}$. Additionally, reductions in subcortical GMV within the hippocampus and amygdala compared to age-matched, healthy controls have also been consistently reported within this population ${ }^{25,26}$. Our findings, obtained from a vastly different population of women with PTSD following IPV-exposure are in contrast with these reports, as we found no differences in CT or GMV when group was dichotomized (PTSD vs control). Interestingly, our findings are consistent with a smaller (but more relevant) literature specifically focusing on homogenous samples of women with PTSD due to physical and sexual abuse. Consistent with our findings, Landre et al. examined a small sample of adult women with PTSD stemming from sexual abuse and found no differences in CT compared to controls ${ }^{37}$. Relatedly, there were no differences in hippocampal volume between women survivors of intimate partner violence and healthy controls ${ }^{38}$. Most recently, it was reported that adolescents with childhood sexual abuse exhibit preserved CT in the ventromedial prefrontal cortex, anterior cingulate gyri, middle temporal gyri, and superior temporal gyri compared to traumafree controls ${ }^{40}$. Collectively, there is growing evidence suggesting that the specific type of trauma exposure (e.g., combat vs. IPV) may explain whether a diagnosis of PTSD predicts alterations in CT and GMV compared to healthy, trauma-free controls.

Although a diagnosis of PTSD did not predict differences compared to healthy controls, specific clusters of PTSD symptoms were associated with CT and GMV within women with IPV-related PTSD. These results suggest that altered brain structure within IPV-exposed women with PTSD may be driven by symptom severity within specific clusters (e.g., re-experiencing, avoidance, hyperarousal), or that these particular alterations led to an increased likelihood of the emergence of more severe symptoms. Moreover, our results revealed that the symptom clusters had a differential effect on brain structure. For instance, greater active avoidance symptoms were associated with greater CT in several regions and greater GMV in the right amygdala, whereas greater reexperiencing and hyperarousal symptoms were associated with decreased CT in several regions and decreased left amygdala volume. The observed differential relationships with the amygdala depending on PTSD symptom clusters is consistent with a recent meta-analysis that found among adults with PTSD, greater re-experiencing symptom severity scores were associated with greater left amygdala activity in response to threat, whereas greater 
A

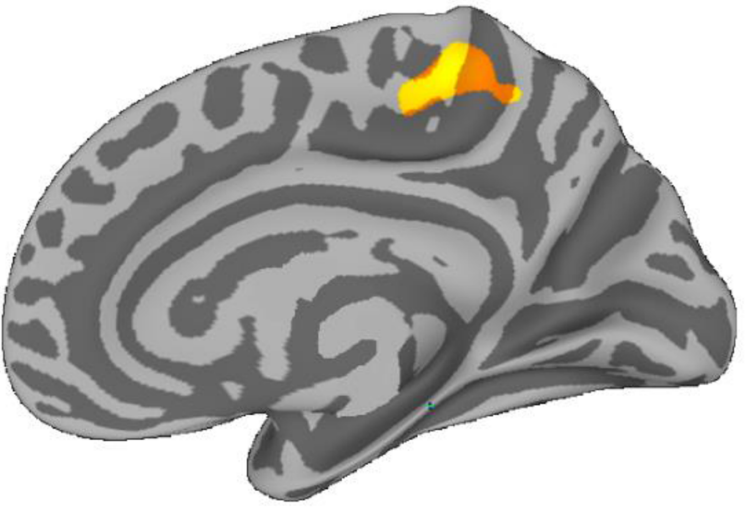

B

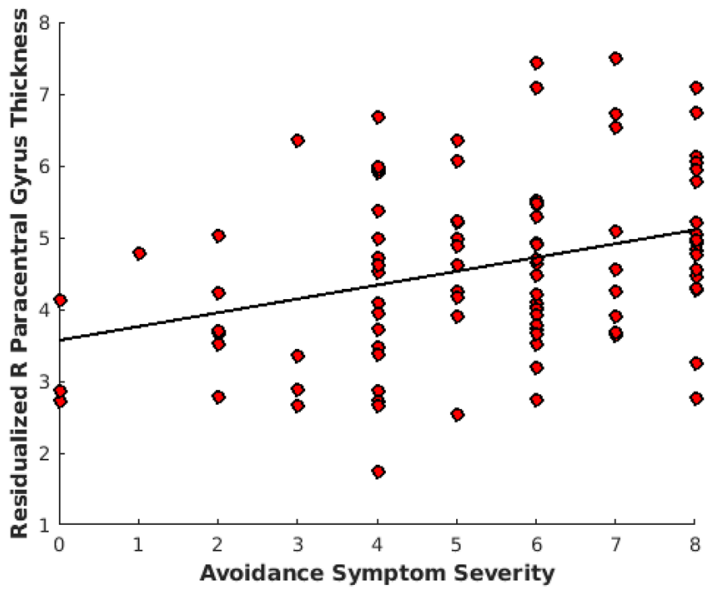

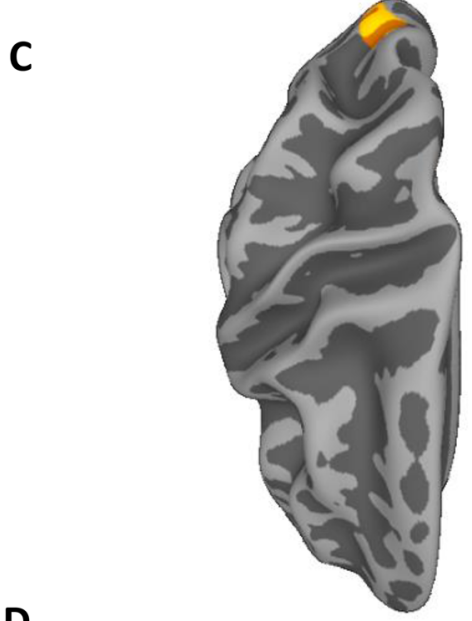

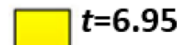

D

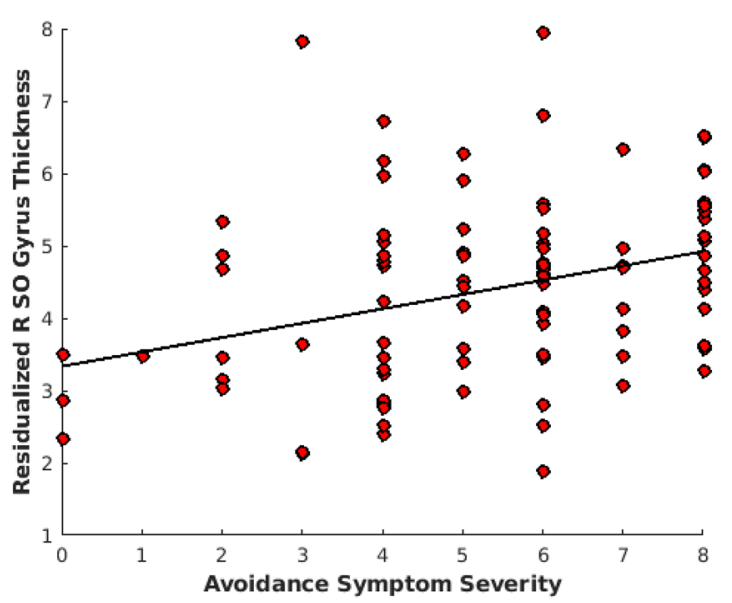

Figure 3. Greater Active Avoidance PTSD symptom severity is associated with increased thickness of the right paracentral, posterior cingulate, and superior occipital gyrus. Vertex-wise linear model tests across the cortical surface revealed two clusters that survived Monte-Carlo correction for multiple comparisons and were associated with greater PTSD re-experiencing symptom severity: the right paracentral and posterior cingulate $(\mathbf{A}, \mathbf{B})$, and superior occipital gyrus (C,D). Active Avoidance symptom severity scores (cluster C; score range of 0-8) were obtained from the PTSD Checklist for DSM-V (PCL-5). PTSD = posttraumatic stress disorder; $\mathrm{R}=$ right; $\mathrm{SO}=$ superior occipital.

avoidance symptom severity scores were associated with less amygdala activity in response to threat ${ }^{78}$. Moreover, a prior investigation reported a positive relationship between avoidance symptoms and right amygdala (and hippocampus) volume, and an inverse relationship between hyperarousal symptoms and left superior medial frontal gyrus volume in a small sample of adults ( $66 \%$ women) with non-combat related PTSD ( $87 \%$ of sample experienced IPV-exposure $)^{39}$.

The differential relationships of PTSD symptom clusters with CT and GMV, in particular regions canonically implicated in PTSD (i.e., amygdala), highlights the importance of acknowledging that 'PTSD' is a diagnostic construct with considerable symptom heterogeneity ${ }^{79}$. Accordingly, researchers may be missing important structural correlates of PTSD in this specific population (women with IPV-exposure) and others (e.g., combat, motor vehicle accidents) by neglecting to examine the effect of each PTSD symptom cluster. For instance, our results revealed decreased CT in the parahippocampal gyrus of adults with greater re-experiencing symptoms. The parrahippocampal gyrus is a region of the cortex that surrounds the hippocampus and plays a role in encoding, and episodic, spatial, and contextual memory. Relatedly, re-experiencing symptoms involve memory retrieval processes. Although investigations are limited, there are two additional reports of decreased parahippocampal volume in PTSD populations (male combat veterans and natural disaster survivors) compared to controls ${ }^{21,23}$. Our findings add to a growing body of evidence suggesting that decreased CT in the parahippocampal gyrus may be a commonly observed structural alteration in PTSD, especially among those with greater re-experiencing symptoms. In contrast, greater avoidance symptoms were associated with greater CT in several regions (left lateral fissure and post central gyrus; right paracentral, posterior cingulate, and superior occipital cortex). Given that CT in these regions did not differ between controls and those with PTSD, further research is warranted to determine whether the observed structural alterations among women with PTSD with greater avoidance symptoms is clinically relevant. Lastly, it is worth noting that the addition of major depressive disorder (MDD) into our 


\begin{tabular}{|c|c|c|c|c|c|c|c|c|}
\hline \multirow[b]{2}{*}{ Predictors } & \multicolumn{2}{|l|}{ Step 1} & \multicolumn{2}{|l|}{ Step 2} & \multicolumn{2}{|l|}{ Step 3} & \multicolumn{2}{|l|}{ Step 4} \\
\hline & t-stat & P value & t-stat & P value & t-stat & P value & t-stat & P value \\
\hline \multicolumn{9}{|l|}{\begin{tabular}{|l|} 
Left amygdala \\
\end{tabular}} \\
\hline Age & -1.02 & .310 & -0.97 & .334 & -1.07 & .288 & -1.13 & .259 \\
\hline Scanner site & -0.38 & .706 & -0.34 & .731 & -0.38 & .703 & -0.58 & .560 \\
\hline TIV & 8.39 & $<.001$ & 8.31 & $<.001$ & 8.31 & $<.001$ & 8.10 & $<.001$ \\
\hline Education & 0.37 & .713 & 0.23 & .814 & 0.27 & .788 & 0.06 & .949 \\
\hline Group & - & - & -0.48 & .631 & 0.07 & .947 & 0.33 & .739 \\
\hline Anxiety & - & - & - & - & -0.90 & .368 & -0.75 & .452 \\
\hline Depression & - & - & - & - & - & - & -1.79 & .076 \\
\hline R-squared & \multicolumn{2}{|l|}{0.412} & \multicolumn{2}{|l|}{0.414} & \multicolumn{2}{|l|}{0.418} & \multicolumn{2}{|l|}{0.434} \\
\hline Adjusted R-squared & \multicolumn{2}{|l|}{0.392} & \multicolumn{2}{|l|}{0.388} & \multicolumn{2}{|l|}{$\mid 0.387$} & \multicolumn{2}{|l|}{0.399} \\
\hline \multicolumn{9}{|l|}{ Right amygdala } \\
\hline Age & -1.32 & .190 & -1.23 & .221 & -1.21 & .229 & -1.30 & .196 \\
\hline Scanner site & -2.45 & .015 & -2.38 & .018 & -2.36 & .019 & -2.64 & .009 \\
\hline TIV & 7.15 & $<.001$ & 7.06 & $<.001$ & 7.03 & $<.001$ & 6.81 & $<.001$ \\
\hline Education & 1.42 & .158 & 1.15 & .254 & 1.14 & .257 & 0.89 & .375 \\
\hline Group & - & - & -0.91 & .366 & -0.80 & .423 & -0.47 & .636 \\
\hline Anxiety & - & - & - & - & 0.07 & .944 & 0.26 & .793 \\
\hline Depression & - & - & - & - & - & - & -2.23 & .027 \\
\hline R-squared & \multicolumn{2}{|l|}{0.362} & \multicolumn{2}{|l|}{0.366} & \multicolumn{2}{|l|}{0.366} & \multicolumn{2}{|l|}{0.393} \\
\hline Adjusted R-squared & \multicolumn{2}{|l|}{0.340} & \multicolumn{2}{|l|}{0.339} & \multicolumn{2}{|l|}{0.333} & \multicolumn{2}{|l|}{0.355} \\
\hline \multicolumn{9}{|l|}{\begin{tabular}{|l|} 
Left hippocampus \\
\end{tabular}} \\
\hline Age & -1.38 & .167 & -1.39 & .164 & -1.23 & .219 & -1.27 & .208 \\
\hline Scanner site & 0.68 & .492 & 0.67 & .504 & 0.73 & .466 & 0.61 & .539 \\
\hline TIV & 9.05 & $<.001$ & 9.01 & $<.001$ & 9.01 & $<.001$ & 8.82 & $<.001$ \\
\hline Education & 1.52 & .132 & 1.52 & .132 & 1.47 & .145 & 1.34 & .181 \\
\hline Group & - & - & 0.22 & .829 & -0.54 & .587 & -0.39 & .696 \\
\hline Anxiety & - & - & - & - & 1.39 & .167 & 1.47 & .144 \\
\hline Depression & - & - & - & - & - & - & -0.98 & .326 \\
\hline R-squared & 0.484 & & 0.484 & & 0.493 & & 0.497 & \\
\hline Adjusted R-squared & 0.466 & & 0.462 & & 0.466 & & 0.466 & \\
\hline Right hippocampus & & & & & & & & \\
\hline Age & -1.43 & .156 & -1.37 & .172 & -1.21 & .227 & -1.25 & .214 \\
\hline Scanner site & 0.55 & .581 & 0.58 & .561 & 0.64 & .523 & 0.52 & .606 \\
\hline TIV & 8.88 & $<.001$ & 8.80 & $<.001$ & 8.80 & $<.001$ & 8.60 & $<.001$ \\
\hline Education & 2.24 & .026 & 2.04 & .043 & 1.99 & .048 & 1.86 & .065 \\
\hline Group & - & - & -0.50 & .618 & -1.14 & .258 & -0.96 & .337 \\
\hline Anxiety & - & - & - & - & 1.35 & .179 & 1.44 & .153 \\
\hline Depression & - & - & - & - & - & - & -1.06 & .290 \\
\hline R-squared & 0.489 & & 0.490 & & 0.498 & & 0.503 & \\
\hline Adjusted R-squared & 0.471 & & 0.468 & & 0.472 & & 0.472 & \\
\hline
\end{tabular}

Table 2. PTSD vs control group as predictor of hippocampus and amygdala gray matter volume. A diagnosis of PTSD did not significantly $(p>.0125)$ predict differences in amygdala (left and right) and hippocampal (left and right) volume. TIV $=$ total intracranial volume.

models yielded some intriguing patterns. For instance, there was an effect for depression to predict left and right amygdala volume in the between group analyses. Though it did not survive correction for multiple comparison, this may suggest that a current diagnosis of MDD, as opposed to PTSD, may be more predictive of amygdala volume in adult women with PTSD. Within the PTSD group, there was also a trend for depression to predict right amygdala volume. Although these findings were not statistically significant after controlling for multiple comparisons, and therefore their inclusion in the models did not influence the interpretation of the PTSD subgroup analyses, they highlight the potential importance of examining mental health comorbidities in addition to PTSD. For instance, Bremner et al. previously reported reduced hippocampal volume in women with PTSD from childhood sexual abuse and a comorbid diagnosis of MDD compared to individuals with current MDD without abuse, but only after controlling for PTSD ${ }^{80}$. While it is plausible that comorbid depression and anxiety may explain unique variance in predicting GMV, that does not appear to be the case for CT, as neither were associated with CT across the cortex in all tested models. As was done in the current study, it may be beneficial 
A

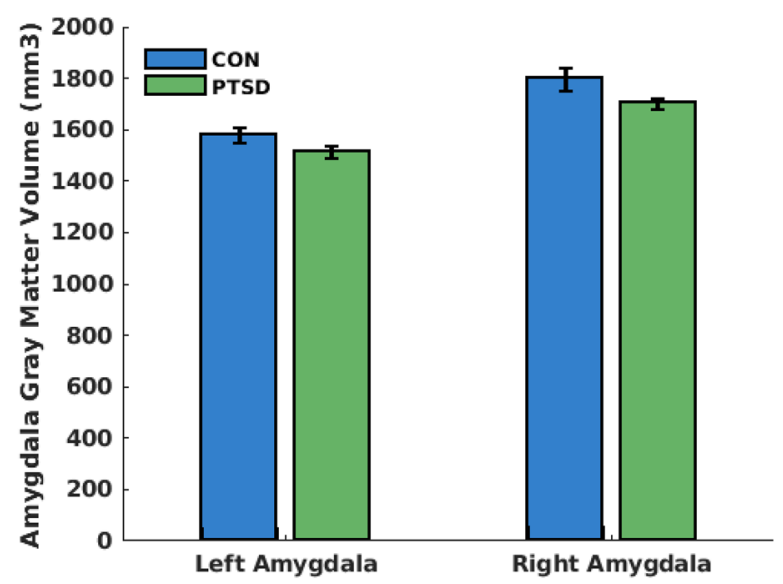

C

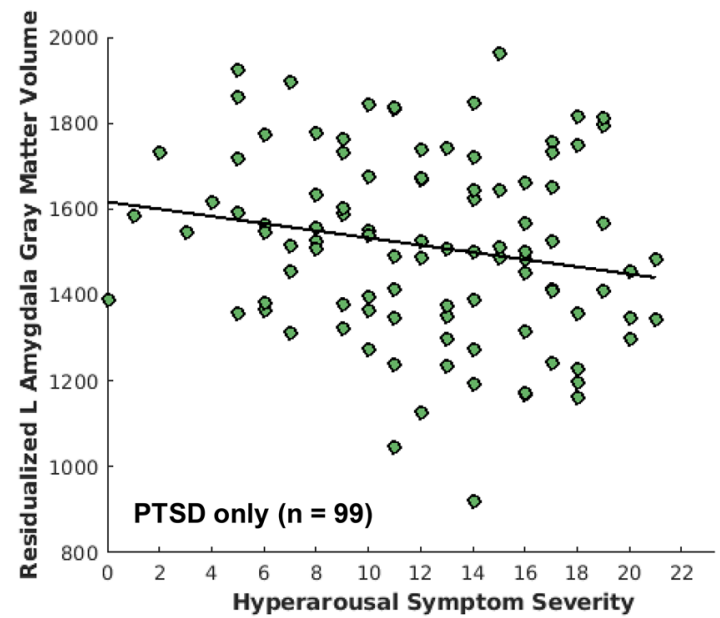

B

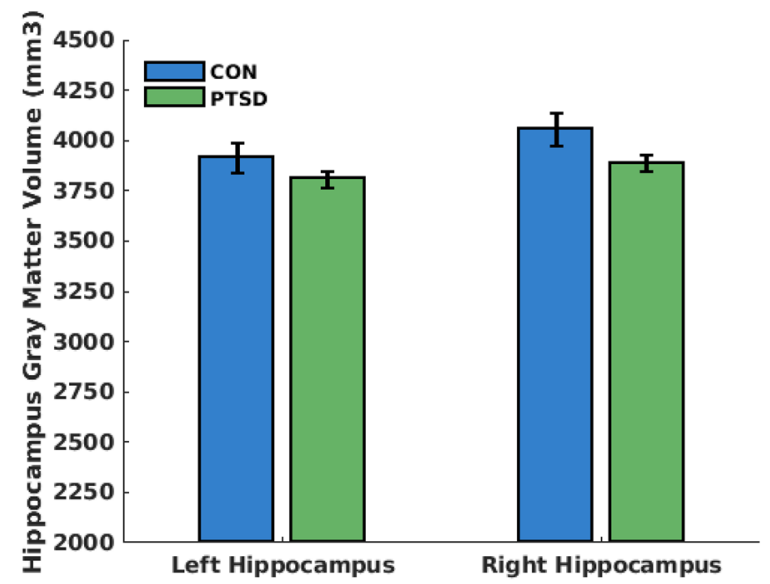

D

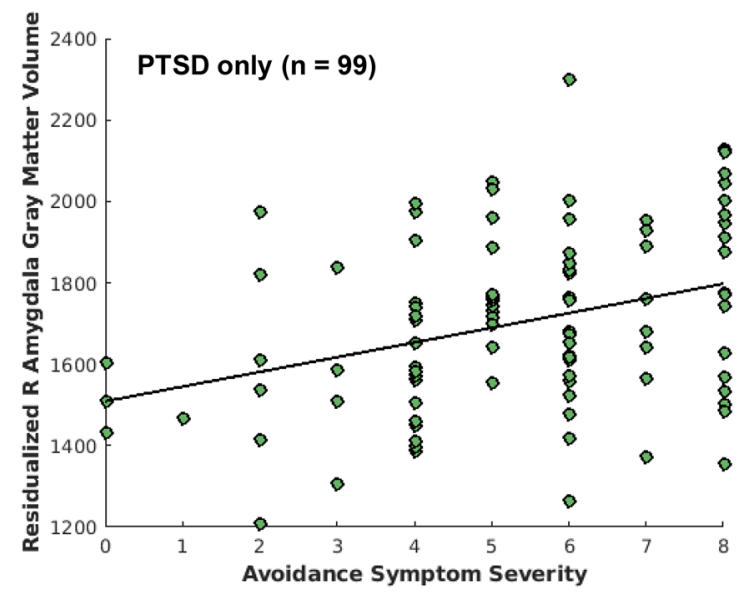

Figure 4. A diagnosis of PTSD in women does not predict differences in amygdala and hippocampal gray matter volume $(\mathbf{A}, \mathbf{B})$. However, within the PTSD group, greater hyperarousal symptoms significantly predicted less left amygdala volume, even after controlling for anxiety and depression (C); whereas greater active avoidance symptoms significantly predicted greater right amygdala volume, even after controlling for anxiety and depression (D). Hyperarousal (cluster E; score range of $0-24$ ) and active avoidance (cluster C; score range of 0 to 8 ) symptom severity scores were obtained from the PTSD Checklist for DSM-V (PCL-5). $\mathrm{PTSD}=$ posttraumatic stress disorder; $\mathrm{L}=$ left; $\mathrm{R}=$ right.

for future research investigations examining PTSD populations to present results with and without comorbid depression and anxiety disorder diagnoses included in statistical models in order to improve our understanding of each diagnoses unique contributions.

This study is notwithstanding limitations. For instance, the sample size of the control group $(n=22)$ was much smaller than that of the PTSD group $(n=99)$, which may potentially explain the lack of group differences in CT and GMV. However, the control group sample size is relatively greater than many of the previous neuroimaging studies that have yielded similar findings ${ }^{36,37}$, and the sample size of the PTSD group $(n=99)$ and overall sample $(\mathrm{n}=121)$ affords greater statistical power than prior studies. Additionally, the control group consisted solely of non-trauma exposed adults, which conflates exposure to trauma with the manifestation of PTSD (for our PTSD group). However, exploratory analyses incorporating trauma exposure as a main predictor yielded similar findings as results obtained from including a dichotomous group classification (i.e., control vs. PTSD). Another potential limitation is that anxiety and depression were simply dichotomized based on whether participants had a current diagnosis (i.e., endorsed diagnostically significant symptoms). The inclusion of depressive and anxiety symptoms on a continuum may have explained more variance for those who were experiencing subthreshold symptoms. Additionally, this was a cross-sectional study, rendering it impossible to determine whether these structural alterations (or lack thereof) were present prior to, or a direct consequence of IPV-exposure and/or the development of PTSD. Moreover, over $80 \%$ of the sample was Caucasian. Although there were no significant differences in race between groups, this highlights the need for additional research with a larger and more racially diverse sample. Lastly, the current study did not track hormonal variations among all participants during the time of their scan, which is a limitation as recent various hormonal factors (e.g., birth control, menstrual cycle stage; sex hormones) are known to influence gray matter volume ${ }^{81-84}$. However, exploratory analyses among the PTSD group (for which data was available) revealed that results examining symptom severity within specific PTSD symptom clusters as a predictor of amygdala and hippocampal GMV did not differ based on whether birth control use was included as a covariate (see Supplementary Table 3). 


\begin{tabular}{|c|c|c|c|c|c|c|c|c|}
\hline \multirow[b]{2}{*}{ Predictors } & \multicolumn{2}{|l|}{ Step 1} & \multicolumn{2}{|l|}{ Step 2} & \multicolumn{2}{|l|}{ Step 3} & \multicolumn{2}{|l|}{ Step 4} \\
\hline & t-stat & P value & t-stat & P value & t-stat & P value & t-stat & P value \\
\hline \multicolumn{9}{|l|}{ Left amygdala } \\
\hline Age & -0.97 & .334 & -0.72 & .474 & -0.82 & .413 & -0.90 & .369 \\
\hline Scanner site & -1.10 & .272 & -0.96 & .340 & -0.99 & .326 & -1.08 & .281 \\
\hline TIV & 8.55 & $<.001$ & 7.55 & $<.001$ & 7.53 & $<.001$ & 7.41 & $<.001$ \\
\hline Cluster B & - & - & -0.08 & .939 & -0.12 & .907 & 0.10 & .923 \\
\hline Cluster C & - & - & 2.13 & .035 & 2.19 & .030 & 1.92 & .058 \\
\hline Cluster D & - & - & 0.07 & .942 & 0.07 & .946 & 0.31 & .757 \\
\hline Cluster E & - & - & -2.84 & .005 & -2.73 & .007 & -2.74 & $.007^{*}$ \\
\hline Anxiety & - & - & - & - & -0.73 & .464 & -0.67 & .506 \\
\hline Depression & - & - & - & - & - & - & -1.05 & .298 \\
\hline R-squared & \multicolumn{2}{|l|}{0.446} & \multicolumn{2}{|l|}{0.523} & \multicolumn{2}{|l|}{0.526} & \multicolumn{2}{|l|}{0.531} \\
\hline Adjusted R-squared & \multicolumn{2}{|l|}{0.429} & \multicolumn{2}{|l|}{0.486} & \multicolumn{2}{|l|}{0.483} & \multicolumn{2}{|l|}{0.484} \\
\hline \multicolumn{9}{|l|}{ Right amygdala } \\
\hline Age & -0.92 & .359 & -0.96 & .342 & -0.93 & .355 & -1.07 & .287 \\
\hline Scanner site & -2.53 & .012 & -2.58 & .011 & -2.56 & .012 & -2.74 & .007 \\
\hline TIV & 7.39 & $<.001$ & 6.11 & $<.001$ & 6.07 & $<.001$ & 5.97 & $<.001$ \\
\hline Cluster B & - & - & -1.17 & .244 & -1.16 & .249 & -0.80 & .424 \\
\hline Cluster C & - & - & 3.02 & .003 & 2.98 & .003 & 2.56 & $.012^{*}$ \\
\hline Cluster D & - & - & -0.15 & .878 & -0.15 & .879 & 0.25 & .803 \\
\hline Cluster E & - & - & -1.46 & .148 & -1.45 & .151 & -1.47 & .144 \\
\hline Anxiety & - & - & - & - & 0.06 & .951 & 0.17 & .865 \\
\hline Depression & - & - & - & - & - & - & -1.71 & .089 \\
\hline R-squared & \multicolumn{2}{|l|}{0.376} & \multicolumn{2}{|l|}{0.452} & \multicolumn{2}{|l|}{0.452} & \multicolumn{2}{|l|}{0.469} \\
\hline Adjusted R-squared & \multicolumn{2}{|l|}{0.357} & 0.409 & & 0.403 & & 0.415 & \\
\hline Left hippocampus & & & & & & & & \\
\hline Age & -0.69 & .491 & -0.58 & .560 & -0.34 & .736 & -0.42 & .678 \\
\hline Scanner site & 0.99 & .322 & 1.22 & .225 & 1.30 & .196 & 1.20 & .234 \\
\hline TIV & 9.91 & $<.001$ & 8.52 & $<.001$ & 8.60 & $<.001$ & 8.48 & $<.001$ \\
\hline Cluster B & - & - & 0.53 & .598 & 0.62 & .538 & 0.81 & .421 \\
\hline Cluster C & - & - & 1.05 & .297 & 0.88 & .381 & 0.64 & .524 \\
\hline Cluster D & - & - & 0.22 & .828 & 0.23 & .819 & 0.46 & .647 \\
\hline Cluster E & - & - & -1.32 & .189 & -1.50 & .136 & -1.51 & .134 \\
\hline Anxiety & - & - & - & - & 1.56 & .121 & 1.63 & .107 \\
\hline Depression & - & - & - & - & - & - & -1.01 & .314 \\
\hline R-squared & 0.541 & & 0.556 & & 0.567 & & 0.572 & \\
\hline Adjusted R-squared & 0.527 & & 0.521 & & 0.529 & & 0.529 & \\
\hline Right hippocampus & & & & & & & & \\
\hline Age & -0.44 & .659 & -0.39 & .699 & -0.16 & .874 & -0.26 & .793 \\
\hline Scanner site & 1.24 & .219 & 1.52 & .131 & 1.60 & .113 & 1.47 & .145 \\
\hline TIV & 9.49 & $<.001$ & 8.00 & $<.001$ & 8.06 & $<.001$ & 7.95 & $<.001$ \\
\hline Cluster B & - & - & 0.63 & .533 & 0.71 & .480 & 0.96 & .338 \\
\hline Cluster C & - & - & 1.03 & .306 & 0.87 & .386 & 0.57 & .573 \\
\hline Cluster D & - & - & -0.32 & .751 & -0.31 & .757 & 0.00 & .996 \\
\hline Cluster E & - & - & -0.48 & .634 & -0.65 & .521 & -0.65 & .515 \\
\hline Anxiety & - & - & - & - & 1.46 & .149 & 1.54 & .126 \\
\hline Depression & - & - & - & - & - & - & -1.32 & .189 \\
\hline R-squared & 0.522 & & 0.533 & & 0.544 & & 0.553 & \\
\hline Adjusted R-squared & 0.507 & & 0.497 & & 0.503 & & 0.508 & \\
\hline
\end{tabular}

Table 3. PTSD symptom clusters as predictors of amygdala and hippocampus grey matter volume. Within the PTSD group, greater hyperarousal symptoms significantly predicted less left amygdala volume, even after controlling for anxiety and depression (denoted ${ }^{\star}$ ); while greater avoidance symptoms significantly predicted greater right amygdala volume, even after controlling for anxiety and depression (denoted ${ }^{\star}$ ). PTSD symptom cluster scores within the PTSD group did not significantly (all ps $>.0125$ ) predict left or right hippocampal volume. TIV = total intracranial volume. 
In conclusion, the current study found no differences in CT or GMV in the amygdala or hippocampus of adult women with PTSD stemming from IPV-exposure, compared to trauma-free women without a diagnosis of PTSD. Although there were no group differences in the current study, re-experiencing, active avoidance, and hyperarousal symptom severity within the PTSD group were significantly associated with differential (increased and decreased depending on the region) CT in several regions and GMV in the amygdala. These findings add to the growing literature suggesting that: (1) the specific type of trauma exposure (e.g., interpersonal violence vs combat) may play an important role in determining whether a diagnosis of PTSD predicts structural brain differences compared to trauma-free controls, (2) alterations in CT and GMV among women with PTSD stemming from IPV-exposure may be driven by symptom severity within specific symptom clusters, as opposed to overall PTSD symptom severity, and (3) symptom severity within PTSD symptom clusters may have a differential effect on brain structure among women with IPV-related PTSD, with greater avoidance symptom severity predicting increased CT and GMV, and greater re-experiencing and hyperarousal symptom severity predicting decreased CT and GMV.

Received: 25 June 2020; Accepted: 28 December 2020

Published online: 19 January 2021

\section{References}

1. APA. Diagnostic and Statistical Manual of Mental Disorders 5th edn. (APA, Washington DC, 2013).

2. Kessler, R. C. Posttraumatic stress disorder: the burden to the individual and to society. J. Clin. Psychiatry 61(55), 4-12 (2000) ((discussion 13-14)).

3. Resnick, H. S., Kilpatrick, D. G., Dansky, B. S., Saunders, B. E. \& Best, C. L. Prevalence of civilian trauma and posttraumatic stress disorder in a representative national sample of women. J. Consult. Clin. Psychol. 61, 984-991 (1993).

4. Kessler, R. C., Sonnega, A., Bromet, E., Hughes, M. \& Nelson, C. B. Posttraumatic stress disorder in the National Comorbidity Survey. Arch. Gen. Psychiatry 52, 1048-1060 (1995).

5. Blanco, C. et al. Toward understanding sex differences in the prevalence of posttraumatic stress disorder: Results from the national epidemiologic survey on alcohol and related conditions. J. Clin. Psychiatry 79 (2018).

6. Breslau, N. et al. Trauma and posttraumatic stress disorder in the community: the 1996 Detroit Area Survey of Trauma. Arch. Gen. Psychiatry 55, 626-632 (1998).

7. Kessler, R. C., Chiu, W. T., Demler, O., Merikangas, K. R. \& Walters, E. E. Prevalence, severity, and comorbidity of 12-month DSM-IV disorders in the National Comorbidity Survey Replication. Arch. Gen. Psychiatry 62, 617-627 (2005).

8. Olatunji, B. O., Cisler, J. M. \& Tolin, D. F. Quality of life in the anxiety disorders: a meta-analytic review. Clin. Psychol. Rev. 27, 572-581 (2007).

9. Resick, P. A., Nishith, P., Weaver, T. L., Astin, M. C. \& Feuer, C. A. A comparison of cognitive-processing therapy with prolonged exposure and a waiting condition for the treatment of chronic posttraumatic stress disorder in female rape victims. J. Consult. Clin. Psychol. 70, 867-879 (2002).

10. Schnurr, P. P. et al. Cognitive behavioral therapy for posttraumatic stress disorder in women: a randomized controlled trial. JAMA 297, 820-830 (2007).

11. Bremner, J. D. et al. MRI-based measurement of hippocampal volume in patients with combat-related posttraumatic stress disorder. Am. J. Psychiatry 152, 973-981 (1995).

12. Hayes, J. P. et al. Automated measurement of hippocampal subfields in PTSD: evidence for smaller dentate gyrus volume. J. Psychiatr. Res. 95, 247-252 (2017).

13. Kasai, K. et al. Evidence for acquired pregenual anterior cingulate gray matter loss from a twin study of combat-related posttraumatic stress disorder. Biol. Psychiatry 63, 550-556 (2008).

14. Pavić, L. et al. Smaller right hippocampus in war veterans with posttraumatic stress disorder. Psychiatry Res 154, 191-198 (2007).

15. Gurvits, T. V. et al. Magnetic resonance imaging study of hippocampal volume in chronic, combat-related posttraumatic stress disorder. Biol. Psychiatry 40, 1091-1099 (1996).

16. Morey, R. A. et al. Amygdala volume changes in posttraumatic stress disorder in a large case-controlled veterans group. Arch. Gen. Psychiatry 69, 1169-1178 (2012).

17. Geuze, E. et al. Thinner prefrontal cortex in veterans with posttraumatic stress disorder. Neuroimage 41, 675-681 (2008).

18. Bing, X. et al. Alterations in the cortical thickness and the amplitude of low-frequency fluctuation in patients with post-traumatic stress disorder. Brain Res. 1490, 225-232 (2013).

19. Wrocklage, K. M. et al. Cortical thickness reduction in combat exposed U.S. veterans with and without PTSD. Eur. Neuropsychopharmacol. 27, 515-525 (2017).

20. Araki, T. et al. Association between lower P300 amplitude and smaller anterior cingulate cortex volume in patients with posttraumatic stress disorder: a study of victims of Tokyo subway sarin attack. Neuroimage 25, 43-50 (2005).

21. Liu, Y., Li, Y.-J., Luo, E.-P., Lu, H.-B. \& Yin, H. Cortical thinning in patients with recent onset post-traumatic stress disorder after a single prolonged trauma exposure. PLoS ONE 7, e39025 (2012).

22. Woodward, S. H. et al. Decreased anterior cingulate volume in combat-related PTSD. Biol. Psychiatry 59, 582-587 (2006).

23. Woodward, S. H., Schaer, M., Kaloupek, D. G., Cediel, L. \& Eliez, S. Smaller global and regional cortical volume in combat-related posttraumatic stress disorder. Arch. Gen. Psychiatry 66, 1373-1382 (2009).

24. Lindemer, E. R., Salat, D. H., Leritz, E. C., McGlinchey, R. E. \& Milberg, W. P. Reduced cortical thickness with increased lifetime burden of PTSD in OEF/OIF Veterans and the impact of comorbid TBI. Neuroimage Clin. 2, 601-611 (2013).

25. Kühn, S. \& Gallinat, J. Gray matter correlates of posttraumatic stress disorder: a quantitative meta-analysis. Biol. Psychiatry 73, 70-74 (2013).

26. O’Doherty, D. C. M., Chitty, K. M., Saddiqui, S., Bennett, M. R. \& Lagopoulos, J. A systematic review and meta-analysis of magnetic resonance imaging measurement of structural volumes in posttraumatic stress disorder. Psychiatry Res. 232, 1-33 (2015).

27. Karl, A. et al. A meta-analysis of structural brain abnormalities in PTSD. Neurosci. Biobehav. Rev. 30, 1004-1031 (2006).

28. Morey, R. A. et al. Fear learning circuitry is biased toward generalization of fear associations in posttraumatic stress disorder. Transl. Psychiatry 5, e700 (2015).

29. Rauch, S. L., Shin, L. M. \& Phelps, E. A. Neurocircuitry models of posttraumatic stress disorder and extinction: human neuroimaging research-past, present, and future. Biol. Psychiatry 60, 376-382 (2006).

30. Wessa, M. \& Flor, H. Failure of extinction of fear responses in posttraumatic stress disorder: evidence from second-order conditioning. Am. J. Psychiatry 164, 1684-1692 (2007).

31. Logue, M. W. et al. Smaller hippocampal volume in posttraumatic stress disorder: a multisite ENIGMA-PGC study: subcortical volumetry results from posttraumatic stress disorder consortia. Biol. Psychiatry 83, 244-253 (2018). 
32. Wang, X. et al. Cortical volume abnormalities in posttraumatic stress disorder: an ENIGMA-psychiatric genomics consortium PTSD workgroup mega-analysis. Mol. Psychiatry https://doi.org/10.1038/s41380-020-00967-1 (2020).

33. Kessler, R. C. et al. Trauma and PTSD in the WHO World Mental Health Surveys. Eur. J. Psychotraumatol. 8, 1353383 (2017).

34. Scott, K. M. et al. Post-traumatic stress disorder associated with sexual assault among women in the WHO World Mental Health Surveys. Psychol. Med. 48, 155-167 (2018).

35. Iverson, K. M. et al. Exposure to interpersonal violence and its associations with psychiatric morbidity in a U.S. National Sample: a gender comparison. Psychol. Violence 3, 273-287 (2013).

36. Breslau, N., Davis, G. C., Andreski, P., Peterson, E. L. \& Schultz, L. R. Sex differences in posttraumatic stress disorder. Arch. Gen. Psychiatry 54, 1044-1048 (1997).

37. Landré, L. et al. Preserved subcortical volumes and cortical thickness in women with sexual abuse-related PTSD. Psychiatry Res. 183, 181-186 (2010).

38. Fennema-Notestine, C., Stein, M. B., Kennedy, C. M., Archibald, S. L. \& Jernigan, T. L. Brain morphometry in female victims of intimate partner violence with and without posttraumatic stress disorder. Biol. Psychiatry 52, 1089-1101 (2002).

39. Weber, M. et al. Voxel-based morphometric gray matter correlates of posttraumatic stress disorder. J. Anxiety Disord. 27, 413-419 (2013).

40. Rinne-Albers, M. A. et al. Preserved cortical thickness, surface area and volume in adolescents with PTSD after childhood sexual abuse. Sci. Rep. 10, 3266 (2020).

41. Kelly, P. A. et al. Cortical thickness, surface area, and gyrification abnormalities in children exposed to maltreatment: neural markers of vulnerability?. Biol. Psychiatry 74, 845-852 (2013).

42. Whittle, S. et al. Childhood maltreatment and psychopathology affect brain development during adolescence. J. Am. Acad. Child Adolesc. Psychiatry 52, 940-952 (2013).

43. Heim, C. M., Mayberg, H. S., Mletzko, T., Nemeroff, C. B. \& Pruessner, J. C. Decreased cortical representation of genital somatosensory field after childhood sexual abuse. Am. J. Psychiatry 170, 616-623 (2013).

44. Sacher, J. et al. Mapping the depressed brain: a meta-analysis of structural and functional alterations in major depressive disorder. J. Affect. Disord. 140, 142-148 (2012).

45. Lorenzetti, V., Allen, N. B., Fornito, A. \& Yücel, M. Structural brain abnormalities in major depressive disorder: a selective review of recent MRI studies. J. Affect. Disord. 117, 1-17 (2009).

46. Bremner, J. D. et al. Hippocampal volume reduction in major depression. Am. J. Psychiatry 157, 115-118 (2000).

47. Brown, T. A., Campbell, L. A., Lehman, C. L., Grisham, J. R. \& Mancill, R. B. Current and lifetime comorbidity of the DSM-IV anxiety and mood disorders in a large clinical sample. J. Abnorm. Psychol. 110, 585-599 (2001).

48. Buckley, T. C., Mozley, S. L., Bedard, M. A., Dewulf, A.-C. \& Greif, J. Preventive health behaviors, health-risk behaviors, physical morbidity, and health-related role functioning impairment in veterans with post-traumatic stress disorder. Mil. Med. 169, 536-540 (2004).

49. Kehle, S. M. et al. Psychiatric diagnoses, comorbidity, and functioning in National Guard troops deployed to Iraq. J. Psychiatr. Res. 45, 126-132 (2011).

50. Kimerling, R., Clum, G. A. \& Wolfe, J. Relationships among trauma exposure, chronic posttraumatic stress disorder symptoms, and self-reported health in women: replication and extension. J. Trauma Stress 13, 115-128 (2000).

51. Ruscio, A. M., Weathers, F. W., King, L. A. \& King, D. W. Male war-zone veterans' perceived relationships with their children: the importance of emotional numbing. J. Trauma Stress 15, 351-357 (2002).

52. Cisler, J. M. et al. Functional reorganization of neural networks during repeated exposure to the traumatic memory in posttraumatic stress disorder: an exploratory fMRI study. J. Psychiatr. Res. 48, 47-55 (2014).

53. Cisler, J. M. et al. Brain and behavioral evidence for altered social learning mechanisms among women with assault-related posttraumatic stress disorder. J. Psychiatr. Res. 63, 75-83 (2015).

54. Ross, M. C., Lenow, J. K., Kilts, C. D. \& Cisler, J. M. Altered neural encoding of prediction errors in assault-related posttraumatic stress disorder. J. Psychiatr. Res. 103, 83-90 (2018).

55. Sartin-Tarm, A., Ross, M. C., Privatsky, A. A. \& Cisler, J. M. Estradiol modulates neural and behavioral arousal in women with posttraumatic stress disorder during a fear learning and extinction task. Biol. Psychiatry. Cogn. Neurosci. Neuroimaging https:// doi.org/10.1016/j.bpsc.2020.04.012 (2020).

56. Kilpatrick, D. G. et al. Violence and risk of PTSD, major depression, substance abuse/dependence, and comorbidity: results from the National Survey of Adolescents. J. Consult. Clin. Psychol. 71, 692-700 (2003).

57. First, M.B. et al. Structured Clinical Interview for DSM-IV-TR Axis I Disorders, Research Version, Non-patient Edition. (SCID-I/ NP). New York, Biometrics Research, New York State Psychiatric Institute. (2002).

58. Weathers, F. W. et al. The Clinician-Administered PTSD Scale for DSM-5 (CAPS-5): development and initial psychometric evaluation in military veterans. Psychol. Assess 30, 383-395 (2018).

59. Blevins, C. A., Weathers, F. W., Davis, M. T., Witte, T. K. \& Domino, J. L. The posttraumatic stress disorder checklist for DSM-5 (PCL-5): development and initial psychometric evaluation. J. Trauma Stress 28, 489-498 (2015).

60. Blanchard, E. B., Jones-Alexander, J., Buckley, T. C. \& Forneris, C. A. Psychometric properties of the PTSD Checklist (PCL). Behav. Res. Ther. 34, 669-673 (1996).

61. Moshier, S. J. et al. An empirical crosswalk for the PTSD checklist: translating DSM-IV to DSM-5 using a veteran sample. J Trauma Stress 32, 799-805 (2019).

62. Fischl, B. et al. Whole brain segmentation: automated labeling of neuroanatomical structures in the human brain. Neuron 33, 341-355 (2002).

63. Reuter, M., Rosas, H. D. \& Fischl, B. Highly accurate inverse consistent registration: a robust approach. Neuroimage 53, 1181-1196 (2010).

64. Dale, A. M., Fischl, B. \& Sereno, M. I. Cortical surface-based analysis I. Segmentation and surface reconstruction. Neuroimage 9 , 179-194 (1999).

65. Sled, J. G., Zijdenbos, A. P. \& Evans, A. C. A nonparametric method for automatic correction of intensity nonuniformity in MRI data. IEEE Trans. Med. Imaging 17, 87-97 (1998).

66. Fischl, B. et al. Automatically parcellating the human cerebral cortex. Cereb. Cortex 14, 11-22 (2004).

67. Dale, A. M. \& Sereno, M. I. Improved localizadon of cortical activity by combining EEG and MEG with MRI cortical surface reconstruction: a linear approach. J. Cogn. Neurosci. 5, 162-176 (1993).

68. Fischl, B., Liu, A. \& Dale, A. M. Automated manifold surgery: constructing geometrically accurate and topologically correct models of the human cerebral cortex. IEEE Trans. Med. Imaging 20, 70-80 (2001).

69. Fischl, B. \& Dale, A. M. Measuring the thickness of the human cerebral cortex from magnetic resonance images. Proc. Natl. Acad. Sci. USA 97, 11050-11055 (2000).

70. Han, X. et al. Reliability of MRI-derived measurements of human cerebral cortical thickness: the effects of field strength, scanner upgrade and manufacturer. Neuroimage 32, 180-194 (2006).

71. Jovicich, J. et al. Reliability in multi-site structural MRI studies: effects of gradient non-linearity correction on phantom and human data. Neuroimage 30, 436-443 (2006).

72. Ségonne, F. et al. A hybrid approach to the skull stripping problem in MRI. Neuroimage 22, 1060-1075 (2004). 
73. Ségonne, F., Pacheco, J. \& Fischl, B. Geometrically accurate topology-correction of cortical surfaces using nonseparating loops. IEEE Trans Med Imaging 26, 518-529 (2007).

74. Klein, A. \& Tourville, J. 101 labeled brain images and a consistent human cortical labeling protocol. Front. Neurosci. 6, 171 (2012).

75. Fischl, B., Sereno, M. I., Tootell, R. B. \& Dale, A. M. High-resolution intersubject averaging and a coordinate system for the cortical surface. Hum. Brain Mapp. 8, 272-284 (1999).

76. Cox, R. W. AFNI: software for analysis and visualization of functional magnetic resonance neuroimages. Comput. Biomed. Res. 29, 162-173 (1996).

77. Qi, S. et al. Cortical inhibition deficits in recent onset PTSD after a single prolonged trauma exposure. Neuroimage. Clin. 3, 226-233 (2013).

78. Chiba, T. et al. A reciprocal inhibition model of alterations between under-/overemotional modulatory states in patients with PTSD. Mol. Psychiatry https://doi.org/10.1038/s41380-020-0827-0 (2020).

79. Galatzer-Levy, I. R. \& Bryant, R. A. 636,120 ways to have posttraumatic stress disorder. Perspect. Psychol. Sci. 8, 651-662 (2013).

80. Bremner, J. D. et al. MRI and PET study of deficits in hippocampal structure and function in women with childhood sexual abuse and posttraumatic stress disorder. Am. J. Psychiatry 160, 924-932 (2003).

81. Lisofsky, N. et al. Hippocampal volume and functional connectivity changes during the female menstrual cycle. Neuroimage 118, 154-162 (2015).

82. Pletzer, B., Harris, T. \& Hidalgo-Lopez, E. Subcortical structural changes along the menstrual cycle: beyond the hippocampus. Sci. Rep. 8, 16042 (2018).

83. Pletzer, B. et al. Menstrual cycle and hormonal contraceptive use modulate human brain structure. Brain Res. 1348, 55-62 (2010).

84. Rehbein, E., Hornung, J., Sundström Poromaa, I. \& Derntl, B. Shaping of the female human brain by sex hormones-a review. Neuroendocrinology https://doi.org/10.1159/000507083 (2020).

\section{Acknowledgements}

Research reported in this publication was supported by the National Institute of Mental Health of the National Institutes of Health under awards MH119132 and MH108753 awarded to Josh M. Cisler. The content is solely the responsibility of the authors and does not necessarily represent the official view of the National Institutes of Health. The funding source had no role in the collection, analysis, and interpretation of data; in writing the report; or in the decision to submit the article for publication.

\section{Author contributions}

K.C. and J.C. wrote the manuscript. K.C. and J.C. designed the study. M.R. and A.S.T. were involved in acquisition of participants. K.C., M.R., A.L., and J.C. were involved in analysis and interpretation of data. All authors reviewed the manuscript.

\section{Competing interests}

The authors declare no competing interests.

\section{Additional information}

Supplementary Information The online version contains supplementary material available at https://doi. org/10.1038/s41598-020-80776-2.

Correspondence and requests for materials should be addressed to K.M.C.

Reprints and permissions information is available at www.nature.com/reprints.

Publisher's note Springer Nature remains neutral with regard to jurisdictional claims in published maps and institutional affiliations.

(c) (i) Open Access This article is licensed under a Creative Commons Attribution 4.0 International License, which permits use, sharing, adaptation, distribution and reproduction in any medium or format, as long as you give appropriate credit to the original author(s) and the source, provide a link to the Creative Commons licence, and indicate if changes were made. The images or other third party material in this article are included in the article's Creative Commons licence, unless indicated otherwise in a credit line to the material. If material is not included in the article's Creative Commons licence and your intended use is not permitted by statutory regulation or exceeds the permitted use, you will need to obtain permission directly from the copyright holder. To view a copy of this licence, visit http://creativecommons.org/licenses/by/4.0/.

(C) The Author(s) 2021 